\title{
Rechargeable Stimulators in Deep Brain Stimulation for Obsessive-Compulsive Disorder: A Prospective Interventional Cohort Study
}

\section{Philippe De Vloo, MD**; Simon Raymaekers, MD*; Kris van Kuyck, PhD+; Laura Luyten, $\mathrm{PhD}^{+\varsigma}$; Lutgardis Gabriëls, MD, PhD; Bart Nuttin, MD, PhD*}

\begin{abstract}
Background: From 1999 onwards, deep brain stimulation (DBS) has been proposed as an alternative to capsulotomy in refractory cases of obsessive-compulsive disorder (OCD). Although rechargeable implantable pulse generators (rIPGs) have been used extensively in DBS for movement disorders, there are no reports on rIPGs in patients with a psychiatric DBS indication, and even possible objections to their use.
\end{abstract}

Objective: We aim to evaluate rIPGs in OCD in terms of effectiveness, applicability, safety, and need for IPG replacement.

Methods: In this prospective before-after study recruiting from 2007 until 2012, OCD patients requiring at least one IPG replacement per 18 months were proposed to have a rIPG implanted at the next IPG depletion. OCD severity was the primary outcome. Ten patients were analyzed.

Results: Psychiatric symptoms and global functioning remained stable in the two years after as compared to the two years before rIPG implantation. Over the same period, the prescribed OCD medication doses did not increase and the DBS stimulation parameters were largely unaltered. Until the end of the follow-up (mean $43 / 4$ years; maximum seven years), the DBS-related surgery frequency decreased and there were no rIPG replacements. During the first few weeks after implantation, two patients obsessively checked the rIPG, but afterwards there were no signs of compulsively checking or recharging the rIPG. Two patients experienced rIPG overdischarges (five occurrences in total).

Conclusions: This is the first report on rlPGs in DBS for OCD patients. The use of rIPGs in this population appears to be effective, applicable, and safe and diminishes the need for IPG replacements.

Keywords: Deep brain stimulation, deep brain stimulation for psychiatric disorders, obsessive-compulsive disorder, rechargeable implantable pulse generator, rechargeable stimulator

Conflict of Interest: Philippe De Vloo, Kris van Kuyck and Laura Luyten have report no competing interests. Medtronic $\circledast$ provided grants for research, education, and traveling for Bart Nuttin and Lutgardis Gabriels. Simon Raymaekers is supported by the Medtronic ${ }^{\circledR}$ Chair for Stereotactic Neurosurgery in Psychiatric Disorders at KU Leuven, held by Bart Nuttin and Lutgardis Gabri els. Bart Nuttin co-owns a patent on DBS in OCD. Parts of this manuscript were presented orally at the 2014 European Society of Stereotactic and Functional Neurosurgery conference in Maastricht, The Netherlands.

\section{INTRODUCTION}

Obsessive-compulsive disorder (OCD) is a psychiatric disorder with a lifetime prevalence of $2 \%$ and is mainly characterized by intrusive thoughts or images (obsessions) and by repetitive or ritualistic actions (compulsions) (1). The current treatment of OCD consists mainly of a combination of selective serotonin reuptake inhibitors [SSRIs] and cognitive behavioral therapy. With this treatment, however, $25-40 \%$ of patients have persistent symptoms and lasting functional impairment. Some of these patients may benefit from neurosurgical treatment such as capsulotomy (2).

From 1999 onwards, deep brain stimulation (DBS) has been proposed as an alternative to capsulotomy in these refractory cases of $\operatorname{OCD}(3,4)$. Clinical trials showed major enhancement of quality of life and dramatic improvement of OCD symptoms in about half of these highly treatment-resistant patients $(5,6)$. In February 2009, the United States Food and Drug Administration (FDA) granted a humanitarian device exemption for DBS in medically refractory OCD. European CE mark approval followed later that year (7). The main targets
Address correspondence to: Philippe De Vloo, MD, Department of Neurosurgery, University Hospitals Leuven, Herestraat 49, 3000 Leuven, Belgium. Email: philippe.devloo@uzleuven.be; philippe.devloo@kuleuven.be

* Department of Neurosurgery, University Hospitals Leuven, Leuven, Belgium;

${ }^{\dagger}$ Research Group Experimental Neurosurgery and Neuroanatomy, KU Leuven, Leuven, Belgium;

₹ Department of Psychiatry, University Hospitals Leuven, Leuven, Belgium; and

$\S$ Research Group Psychology of Learning and Experimental Psychopathology, KU Leuven, Leuven, Belgium

For more information on author guidelines, an explanation of our peer review process, and conflict of interest informed consent policies, please go to http:// www.wiley.com/WileyCDA/Section/id-301854.html

Sources of financial support: This work was supported by the Research Foundation - Flanders (FWO) grants G0A5513N and G072929. Medtronic generously provided all rechargeable devices, but had no influence in the decision to undertake this study, study design, data collection, data analysis, manuscript writing or decision to submit the manuscript. 
currently used for DBS in OCD are the anterior limb of the internal capsule, the ventral striatum (including the bed nucleus of the stria terminalis $[\mathrm{BNST}])$, the nucleus accumbens and the subthalamic nucleus (8-10).

The non-rechargeable nature of implantable pulse generator (IPG) batteries has necessitated regular revision surgery until rechargeable IPGs (rIPGs) were introduced and FDA and CE approved, first in spinal cord stimulation (in 2004) and later in DBS in Parkinson's disease and essential tremor (in 2009) $(11,12)$.

Although rIPGs in DBS for OCD patients have been used in at least two cases $(8,13)$, to the best of our knowledge there are no thorough reports on the use of rIPGs in this highly specific population. Prudence on using rIPGs in DBS for OCD has been called for, usually based on one or more of the following three arguments. First, one could fear obsessions and compulsions regarding battery recharging and checking (8). Secondly, the use of a rIPG requires a strict recharging schedule, which might be interrupted accidently or due to a lack of motivation in depressed patients. Of note, acute IPG depletion can lead to a sudden emergence of depression, anxiety, and even suicidality $(14,15)$. Lastly, one might argue that regular IPG replacements necessitate a strict and scheduled follow-up, which can be beneficial in this psychiatric population, and that patients with a rIPG could get lost to follow-up (ESSFN 2014 Meeting Presentation, R. Schuurman).

The aim of this study is to share our experience with rIPGs in DBS for OCD patients.

\section{MATERIAL AND METHODS}

\section{Patient Selection}

In 2007, a prospective study (ClinicalTrials.gov identifier NCT02685280), approved by the Leuven University Hospitals Ethics Committee, was initiated. DBS for OCD patients were proposed rIPG implantation if they had a beneficial effect of DBS on the psychiatric symptoms and needed at least one IPG replacement per 18 months. The advantages, disadvantages, risks, and practical modalities of using rIPGs were discussed extensively at the outpatient clinic, and patients were allowed to think about their decision for at least one month. Our inclusion criteria for DBS treatment and surgical target details have been described elsewhere (10).

Twelve patients were included between October 1, 2007 and December 31, 2012, two of which were excluded from analysis due to their simultaneous inclusion in the yet unpublished Reclaim Study (ClinicalTrials.gov identifier NCT01135745). Only one patient who met the inclusion criteria refused participation, because she and her relatives considered herself incapable of recharging regularly due to unfamiliarity with modern technical devices. Psychiatric assessment, stimulation parameters, recharging information, pharmacotherapy, hospitalization days, and outpatient clinic visiting frequency were collected from two years before to two years after rIPG implantation, while the number of surgical procedures and adverse events (AEs) were recorded from first DBS electrode implantation until December 31, 2014. See supplementary methods for patient labeling.

\section{rIPG Implantation}

The surgical procedure consisted of removal of both (depleted) non-rechargeable IPGs (nrIPG), tunneling of the extension wires to one of the existing subcutaneous pockets and implantation of a single rIPG. All IPGs were manufactured by Medtronic (Minneapolis, MN, USA). Details can be found in the Supporting Information Table S1.

\section{Training}

The rIPG was recharged for the first time during the hospitalization following the implantation under the guidance of the psychiatrist or DBS technician. During the first weeks after rIPG implantation, outpatient clinic visits were scheduled almost weekly and there was an open-door policy to overcome potential recharging difficulties.

\section{Psychiatric Scales}

Yale-Brown Obsessive Compulsive Scale (Y-BOCS) (16), Hamilton Anxiety Rating Scale (HAM-A) (17), Hamilton Depression Rating Scale (HAM-D) (18), Beck Depression Inventory (BDI) (19), and Global Assessment of Functioning (GAF) (20) were recorded at least once per six months. For statistical analysis, these values were averaged if they were recorded more than once per six months.

\section{Stimulation Parameters}

All reported parameters are the values as programmed with the Medtronic N'Vision physician programming device (N'Vision).

\section{Medication}

To rate OCD medication, we used four classes: SSRIs, selective noradrenalin reuptake inhibitors (SNRIs), tricyclic antidepressants (TCAs), and benzodiazepines (BZDs). Per medication class, prescribed medication was expressed as daily equivalent dose (DED). Details can be found in the supplementary methods.

\section{Recharging Data}

Recharging data (frequency, duration, and typical coupling) were obtained from the N'Vision. These values were recorded for each patient at least once per six months. If these parameters were recorded more than once per six months, the recorded values were averaged. "Typical coupling" refers to the quality of coupling between the recharger's antenna and the rIPG on an eight-step scale, with a higher number indicating better positioning and faster, more efficient recharging (21).

\section{Design}

For this prospective interventional study, a quasi-experimental before-after design was preferred above a randomized controlled trial, both for ethical reasons and because blinding the patient is very hard due to the need for recharging. The primary outcome was defined as the change in Y-BOCS after rIPG implantation.

\section{Statistics}

Applied statistics were paired $t$-tests with Bonferroni correction for comparing mean psychiatric scores, stimulation parameters and surgery frequency. Interrupted time series (ITS) analysis was performed for Y-BOCS. For comparing medication equivalent doses, outpatient clinic visiting frequency and hospitalization periods, related samples Friedman's 2-way ANOVA by ranks was used, with the related samples Wilcoxon signed rank test as post-hoc analysis. $P<0.05$ was considered statistically significant. Calculations were performed with IBM SPSS Statistics 22.0 (Armonk, NY, USA).

\section{RESULTS}

\section{Demographics}

Patient characteristics (five male, five female) are shown in Table 1. 
Table 1. Patient Characteristics.

Patient characteristics

Age at rIPG implantation

Y-BOCS before first DBS electrode implantation

Time since first nrIPG implantation at rIPG implantation

Total number of used nrIPG batteries* at rIPG implantation

Longevity of all used nrIPGs before rIPG implantation

Longevity of last used nrIPG before rIPG implantation

Follow-up since rIPG implantation

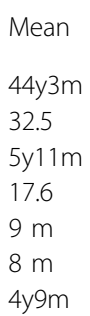

Mean
$44 y 3 \mathrm{~m}$
32.5
$5 \mathrm{y} 11 \mathrm{~m}$
17.6
$9 \mathrm{~m}$
$8 \mathrm{~m}$
$4 \mathrm{y} 9 \mathrm{~m}$

Minimum
$28 y 5 m$
30
$2 y 5 m$
4
$3 m$
$3 m$
$3 y 2 m$

Maximum

$63 y 7 m$

36

$10 \mathrm{y} 1 \mathrm{~m}$

42

$45 \mathrm{~m}$

$18 \mathrm{~m}$

$7 y 2 m$

*nrIPGs were always implanted bilaterally and replaced bilaterally, except in patient\#4, who was mostly stimulated unilaterally.

$y, y e a r(s) ; m$, month(s).

\section{Psychiatric Assessment}

In the two years before and after rIPG implantation, mean Y-BOCS was 14.3 vs. $14.3(P=0.99)$. ITS analysis showed no significant trend changes $(1.35 ; P=0.20)$ nor step changes $(0.193 ; P=0.93)$ at $\mathrm{rIPG}$ implantation. Comparing the same periods, changes in HAM-A (10.7 vs. 11.0$)$, HAM-D (10.2 vs. 9.7), BDI (13.5 vs. 14.8), and GAF (72.0 vs. 71.0) were not statistically significant either $(P=0.75 ; 0.64 ; 0.40$, and 0.43 , respectively) (Fig. 1). Six of our patients spontaneously mentioned at the outpatient clinics that the rIPG had diminished the uncertainty intrinsic to the nrlPGs and the fluctuations that came with the battery depletions and replacement procedures. All patients declared to be happy with the rIPG.

\section{Stimulation Parameters}

The programmed amplitude was lower during the two years before vs. the two years after rIPG implantation (7.22 vs. 7.34V on the right $[P<.01]$ and 6.37 vs. $6.41 \mathrm{~V}$ on the left $[P<0.01])$. Noteworthy, there was a significantly lower voltage programmed on the left as compared to the right side during the assessed four years (6.39 vs. $7.27 \mathrm{~V} ; P<0.001)$. After excluding patient\#4, who was mainly stimulated unilaterally, the difference was smaller (7.08 vs. $7.19 \mathrm{~V}$; $P<0.001)$. On the day of implantation, the rIPG amplitude was programmed lower than the last nrlPG programmed amplitude (mean voltage 6.51 vs. $7.29 \mathrm{~V} ; P=0.018$ ). Average programmed stimulation frequency and pulse width during the two years before and after rIPG implantation were 126.0 vs. $126.3 \mathrm{~Hz}$, respectively $(P=0.02)$ and 291.1 vs. $291.2 \mu \mathrm{sec}$, respectively ( $P=0.82$ ) (Fig. 2).

\section{Medication}

Except for TCAs (0.45 vs. 0.27 DEDs; $P=0.03$ ), no significant changes in prescribed DEDs during the two years before vs. after rIPG implantation were observed (SSRI: 0.85 vs. 0.56 DEDs; $P=0.30$;
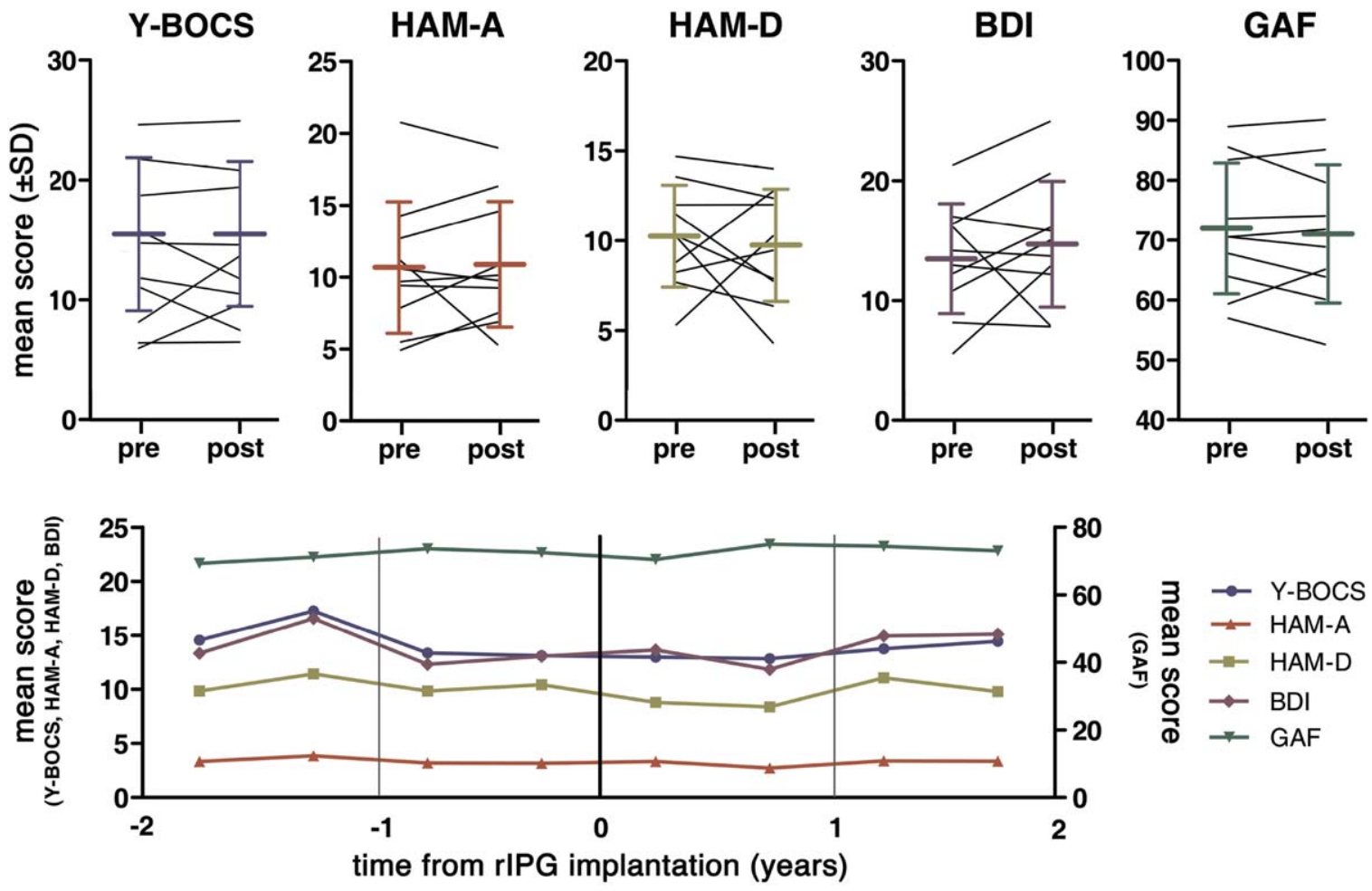

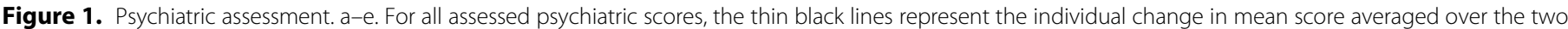

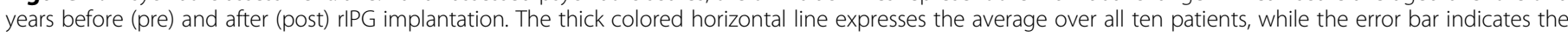

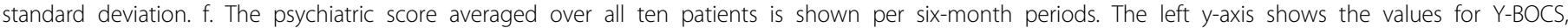

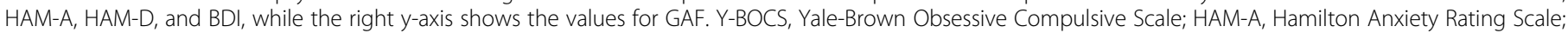
HAM-D, Hamilton Depression Rating Scale; BDI, Beck Depression Inventory; GAF, Global Assessment of Functioning. 


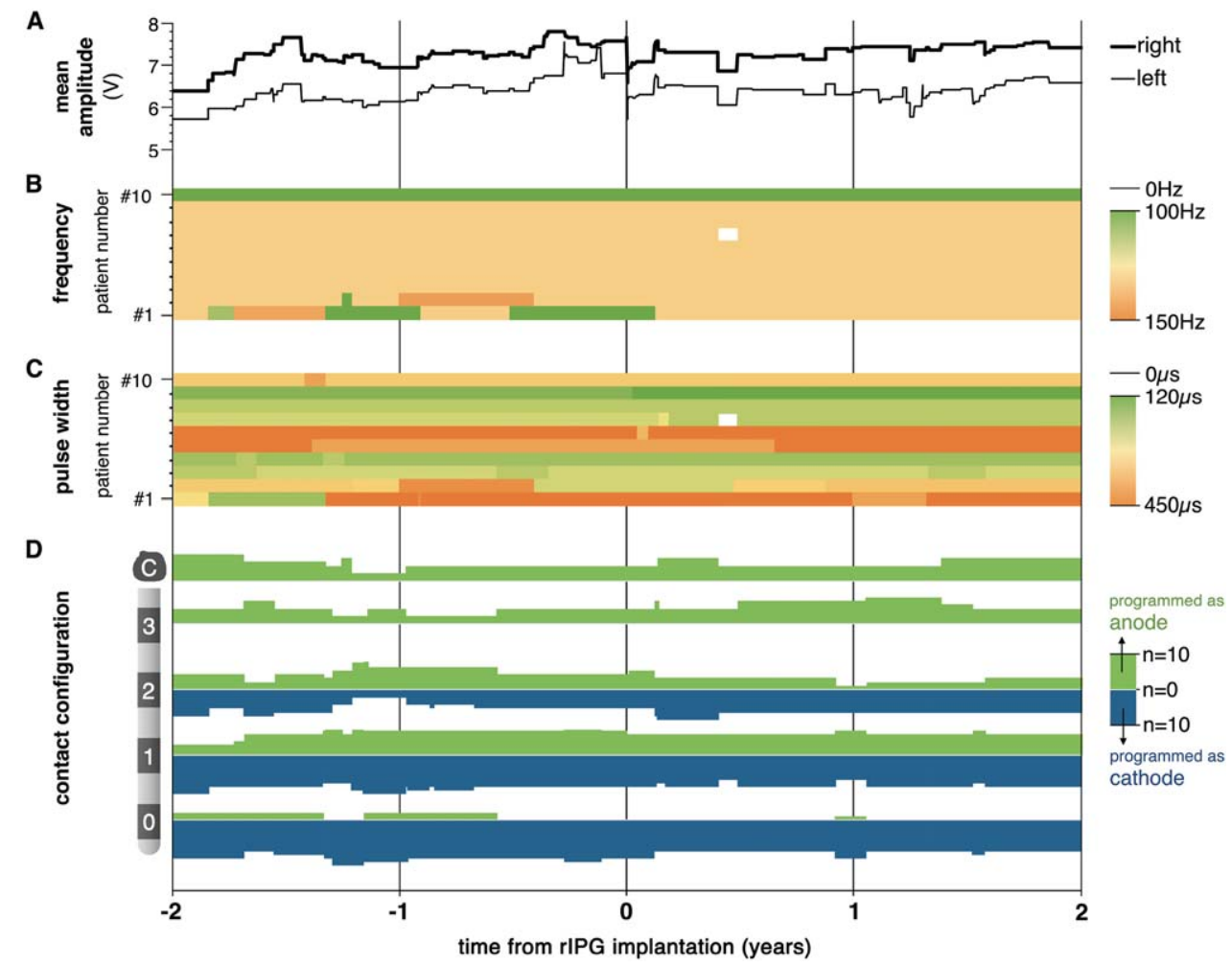

Figure 2. Stimulation parameters. Mean programmed amplitude, frequency, pulse width, and contact configuration are shown over a period ranging from two years before to two years after rIPG implantation. a. Programmed amplitude on the right sided electrode (thick line) and left sided electrode (thin line) are averaged over all ten patients. b-c. Frequency and pulse width are shown with each row representing one patient. The used color indicates the programmed frequency or pulse width, with increasing values from green to red. White represents a value of 0 (stimulation off). $\mathrm{d}$. Contact configuration. Contacts are numbered from 0 (most ventral contact) to 3 (most dorsal contact). "C" represents the IPG case. Per contact number and per day, the amount of contacts being programmed as anode (green) or cathode (blue) is expressed as a bar chart. In bilaterally stimulated patients, there are at least two cathodes and one anode per patient. In one electrode lead, multiple contacts can be programmed as anode or cathode.

SNRI: 0.33 vs. 0.30 DEDs; $P=0.41$; BZD: 1.18 vs. 0.79 DEDs; $P=0.58)$ (Fig. 3a). Only one patient switched between two SSRI types. One patient was not prescribed any OCD medication during the assessed four years.

\section{Outpatient Clinic Visits and Hospitalizations}

Between six months and two years after rIPG implantation, the mean annual combined Psychiatry and Neurosurgery consultation frequency was lower than during the two years before rIPG implantation and than during the first six months after rIPG implantation (5.8 vs. 10.4 visits; $P<0.01$ and 5.8 vs. 8.3 visits; $P=0.047$, respectively) (Fig. 3b). Similarly, between six months and two years after rIPG implantation, the mean annual combined Psychiatry and Neurosurgery hospitalization duration was lower than during the two years before rIPG implantation and than during the first six months after rIPG implantation ( 0.6 vs. 2.9 days; $P=0.036$ and 0.6 vs. 4.2 days; $P<0.01$, respectively) (Fig. 3b). Neurosurgical and Psychiatric hospitalizations were all due to DBS-related surgery and psychiatric decompensation, respectively. During the first six months after rIPG implantation, $85 \%$ of the hospitalization days were due to rIPG implantation surgery recovery. There was no significant difference between the mean hospitalization duration for recovery after the last nrIPG vs. rIPG implantation (1.2 vs. 1.8 days; $P=0.08$ ).

\section{Surgical Procedures}

Before rIPG implantation, average annual DBS-related surgery frequency (excluding the initial electrode implantation) was higher than after rIPG implantation (1.4 vs. 0.04; $P<0.001)$. There were on average 1.3 IPG replacement procedures per year before rIPG implantation, while over the full length of the follow-up period no rIPG needed to be replaced $(P<0.01)$ (Fig. 4).

\section{Recharging}

Recharging parameters are indicated in Table 2.

\section{Adverse Events}

\section{Surgical Complications}

Two surgical procedures were performed after rIPG implantation. Patient\#6 needed a surgical revision one year and five months after rIPG implantation due to an imminent rIPG skin perforation. The surgical procedure consisted of creation of a new subcutaneous pocket with a thicker layer of overlying tissue. Patient\#8 needed a unilateral electrode and extension wire revision due to breakage as discovered through very high impedance over one electrode contact.

There were no surgical infections from the first DBS electrode implantation until the end of follow-up.

\section{Recharging}

For an overview of recharging associated AEs, see supplementary Figure $\mathrm{S} 1$. The most common AE directly associated with recharging was recharger belt breakage. In total, there were nine belt breakages in six patients (i.e., one breakage per $5 \mathrm{y} 3 \mathrm{~m}$ follow-up).

Two patients and their relatives mentioned compulsions or obsessions with regard to recharging during the first weeks after rIPG 

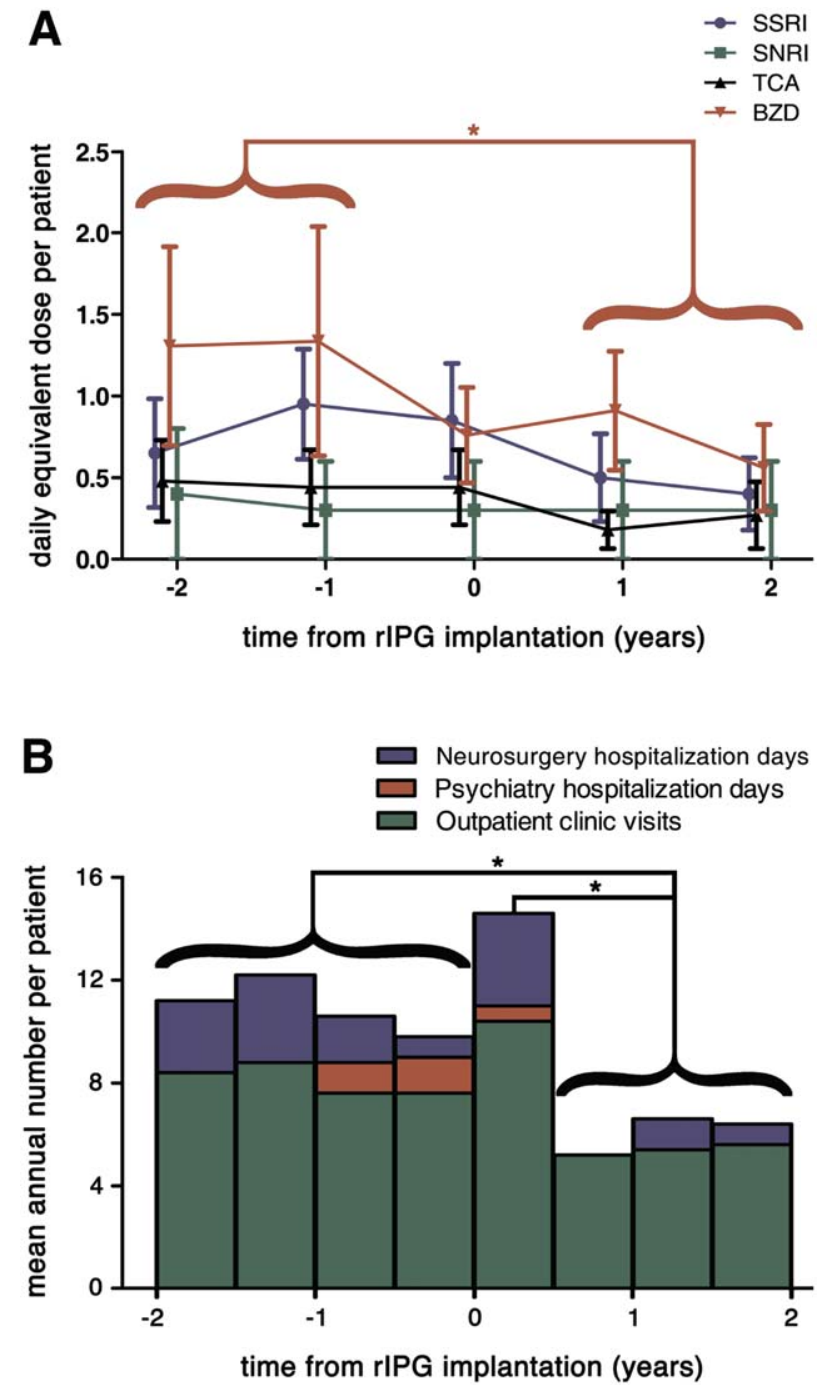

Figure 3. Prescribed OCD medication and Neurosurgery and Psychiatry outpatient clinic visits and hospitalization days. a. From two years before to two years after rIPG implantation, prescribed OCD medication DEDs per medication class, averaged over all ten patients, are expressed as mean \pm standard deviation. b. From two years before to two years after rIPG implantation, the number of outpatient clinic visits (green), and the number of hospitalization days at the Neurosurgery (blue) and Psychiatry (red) ward are displayed. * represents $P<0.05$. SSRIs, selective serotonine reuptake inhibitors; SNRI, selective noradrenalin reuptake inhibitors; TCA, tricyclic antidepressants; and BZD, benzodiazepines.

implantation. In these patients, we advised to recharge once daily, regardless of the battery status, and programmed a daily alarm tone as a reminder. This quickly solved the problem. Based on the N'Vision read-outs and the information provided by the patient and his relatives, this behavior never reappeared. Later, one other patient mentioned the need to recharge to $100 \%$ loading status at each recharging session. Over all read-outs in all patients, the lowest recharging interval was 0.7 days.

Recharger antenna-rIPG coupling problems occurred very early after rIPG implantation in two patients. This problem resolved spontaneously when the patients became acquainted with the system. Coupling problems recurred later in patient\#7, who had experienced a $40 \mathrm{~kg}$ weight loss (body mass index drop from 35 to $24 \mathrm{~kg} / \mathrm{m}^{2}$ ) over the first six months following bariatric surgery. The skin overlying the rIPG had become very loose, making it hard to obtain a stable position of the recharger's antenna in relation to the rIPG. With education, the patient learned how to achieve good coupling in this new situation.

Before rIPG implantation, there were in total 19 complete (IPG read-out no longer possible) nrIPG depletions in six of our patients. These depletions were always associated with psychiatric deterioration. After rIPG implantation, two patients experienced overdischarges (complete rIPG battery depletions). Patient\#1 had a total of three overdischarges: one due to not recharging the recharging device without clear reason, one due to a broken recharger, and one due to intentionally not recharging during a gastro-enteritis, from fear of worsening the stomachache. All were associated with sudden OCD symptom worsening, for which the patient requested an urgent appointment at the Psychiatry department, where the overdischarges were detected and easily resolved by a regular recharging session. Patient\#10 had two overdischarges, both due to not recharging during episodes of severe psychiatric worsening, the second time even during hospitalization at an external psychiatric hospital. The first time, the rIPG could be recharged after performing a physician charging session, which is a charging session performed by the physician, imperative when the battery had been depleted long-lasting (21). After the second overdischarge, we proposed to attempt a second physician charging session, if this would turn out to be impossible, we would propose the patient to replace the rIPG by a nrIPG or to perform a capsulotomy.

None of our patients mentioned a heating sensation or local skin irritation during recharging, although patient\#3 reported a strange indescribable abdominal feeling while recharging. This feeling was only present for five weeks, after which recharged trouble-free again.

Other

Other AEs mentioned by the patients during consultations and hospitalizations at all departments in our hospital are summarized in supplementary Table S2. Interestingly, while half of the patients complained of memory defects, objective memory deficits could never be detected during neuropsychological testing.

\section{DISCUSSION}

\section{Efficacy}

We found no evidence for changes in efficacy of DBS for OCD after switching to a rIPG. No significant changes were noted for all

Table 2. Recharging Parameters.

\begin{tabular}{|c|c|c|c|c|}
\hline Time from rIPG implantation & $0-6 \mathrm{~m}$ & $7-12 m$ & $13-18 m$ & $19-24 m$ \\
\hline Mean recharging frequency & $1 \times / 2.2 \mathrm{~d}$ & $1 \times / 1.4 \mathrm{~d}$ & $1 \times / 1.5 \mathrm{~d}$ & $1 \times / 1.3 \mathrm{~d}$ \\
\hline Mean reacharging duration (h) & 1.8 & 1.7 & 1.3 & 1.4 \\
\hline Mean typical coupling (/8) & 7.1 & 7.8 & 7.9 & 8.0 \\
\hline
\end{tabular}




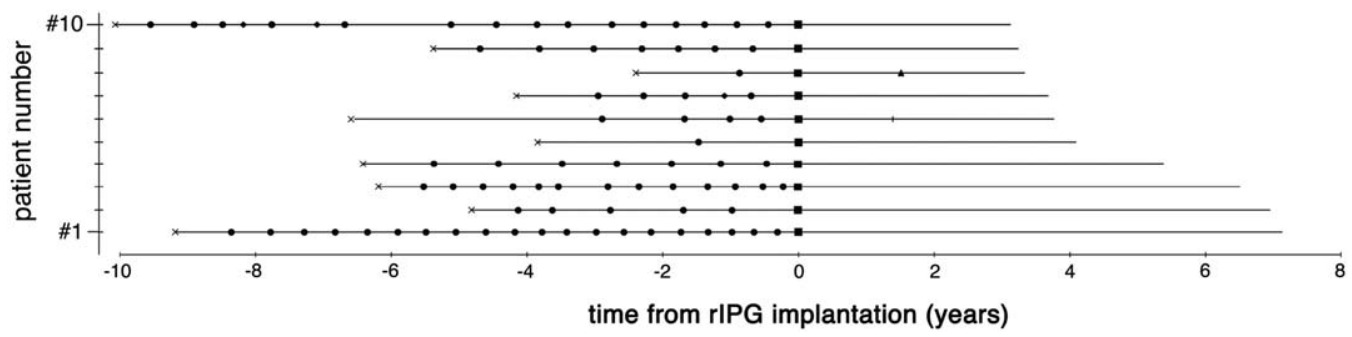

Figure 4. OCD-related surgery timeline. All DBS for OCD-related operations were listed and expressed in a timeline. Each horizontal bar represents one patient (\#1 on bottom row, \#10 on top row). The length of the bar shows the period from first implantation of the electrodes (marked " $x$ ") until last follow-up. IPG replacement for (impending) battery depletion is marked "“", extension wire revision is marked " $\bullet$ ", revision of the abdominal pocket to create a thicker overlying tissue layer is marked "|" and revision of one of the electrodes is marked " $\mathbf{\Delta}$." The implantation of the rIPG is marked "

assessed psychiatric scores including Y-BOCS and no Y-BOCS step or trend changes were observed at rIPG implantation on ITS analysis. Although the need for regular recharging demands attention, the global assessment of social, occupational, and psychological functioning (GAF score) remained stable as well.

To prevent possible overstimulation by the new device and since the amplitude is usually programmed higher in nearly depleted IPGs to compensate for the decreasing battery voltage, the mean rIPG amplitude was programmed lower immediately after implantation as compared to the last programmed amplitude of the nrIPG (6.51 vs. $7.29 \mathrm{~V})$. Afterwards, the programmed amplitude was gradually increased, reaching on average $6.70 \mathrm{~V}$ and $6.93 \mathrm{~V}$ one and two months later, respectively (Fig. 2). Programmed voltage fluctuated over the assessed period. Although statistically significant, these small changes $(<0.25 \mathrm{~V}$ or $<3.5 \%$ difference between the mean annual amplitudes) are probably not clinically relevant. For the programmed stimulation frequency, the same conclusion can be drawn, with the minimal change in stimulation frequency $(0.3 \mathrm{~Hz})$ not even being programmable in an individual patient. A similar initial reduction in programmed stimulation parameters followed by a gradual increase to a level comparable to that of a few months before rIPG implantation was observed in a study in mainly motor disorder patients (22).

Both before and after rIPG implantation, the most frequently programmed contact configuration was bipolar, with contact 0 as anode and contact 1 as cathode. This is a direct consequence of the applied electrode implantation technique and target definition, aiming to position contact 0 in the BNST (10). Importantly, there were no apparent changes in contact configuration after rIPG implantation.

Likewise, none of the prescribed psychiatric medication doses were increased, and average prescribed TCA DED was even decreased, indicating that the stable psychiatric scores were not obtained by increased medication intake.

Both the combined Neurosurgery and Psychiatry outpatient clinic visiting frequency and hospitalization time was lower between six months and two years after rIPG implantation as compared to before rIPG implantation, preceded by an initial increase during the first six months after rIPG implantation. For outpatient clinic visits, this increase is at least partly due to the fact that these patients were invited to the outpatient clinic very frequently in the first month after rIPG implantation, for timely detection of possible recharging problems. For rIPG implantation, our patients were hospitalized during 1.8 days on average. This hospitalization duration does not differ from that for implantation of the last pair of nrlPGs, but due to the applied processing method these hospitalization days are now clustered together in the same time frame, creating an apparent increase in the hospitalization duration in the first six months after rIPG implantation.

Note that hospitalizations at external hospitals could not be assessed reliably and were therefore not taken into account.

\section{Applicability}

Although patient satisfaction with rIPGs in DBS for movement disorders is generally high $(22,23)$, it has been questioned whether rIPGs are applicable in an OCD population (8). Generally, there have been three possible objections put forward, which we can refute to a large extent.

\section{Compulsively and Obsessively Recharging}

One could fear that OCD patients with a rIPG could become obsessed with recharging and checking the battery status and/or could compulsively do so (8). The mean recharging interval in our patients is lower than the interval reported in patients with electrical stimulation for movement disorders and pain (12), presumably with different battery consumption compared to our series. The reported mean recharging duration after six months (108 min/session) however, is very comparable to our series (12).

Over time there seems to be a slow decrease in recharging interval and duration. However, apart from the first few weeks after rIPG implantation, no patient reported recharging obsessions or compulsions, nor was this observed in N'Vision read-outs. As the Y-BOCS remained stable after rIPG implantation, there is no indication that the need for recharging itself might lead to an increase in obsessive or compulsive symptoms.

\section{Recharging Difficulties With Possible Overdischarging}

Acute cessation of DBS for OCD, e.g., in case of IPG depletion, can potentially lead to the emergence of depression, anxiety, and suicidality (15). There is a case report of a patient experiencing a rIPG overdischarge with a full rebound of severe $O C D$, depression and suicidality within 24 hours (13). Moreover, it is considered uncertain whether OCD patients with a depressive mood are capable of recharging the rIPG punctually.

In our series, all patients were capable of learning how to recharge correctly, with excellent typical coupling from the beginning (7.1/ 8 during the first six months) raising to perfect (mean 7.9/8 and 8/8) coupling after one year. Nevertheless, two patients had five battery overdischarges in total. However, one should keep in mind that also in current nrIPGs, predicting the moment of battery depletion is very difficult (24). In our series, the incidence of rIPG overdischarging was even lower than that of complete nrIPG depletion (5 per 17287 follow-up days, i.e., once per nine follow-up years vs. 19 per 21559 follow-up days, i.e., once per three follow-up years; $P=0.019$ ).

\section{Loss to Follow-Up}

Some authors state that the need of nrIPG replacement obliges the patient to regularly attend follow-up consultations, which might be beneficial for these patients, and that this need could be decreased in patients having rIPGs. Although a significant decrease in outpatient clinic contacts is observed from six months following 
rIPG implantation onwards, no patients were lost to follow-up. On the contrary, this effect can be considered as an advantage in terms of self-confidence and control, as mentioned by most of our patients. O'Rawe also states that the rechargeable nature of the rIPG has been reassuring for their patient, as he was able to exert control over his battery life (13).

\section{Safety}

Both surgical complications, being an imminent skin perforation and a broken electrode, required surgical re-intervention. We consider none of the AEs, except for recharger belt breakage, as being typically associated with rIPGs.

In the literature, two surgical complications associated specifically with the use of rIPGs have been described. First, in a patient with DBS for Parkinson's disease, recharging difficulties were noticed after the rIPG had flipped upside down, necessitating surgical reorientation, and fixation (25). Secondly, two cases of the so-called "shielded battery syndrome" have been published, in which the additional pocket adaptor needed for replacing certain types of nrIPGs to rIPGs migrated superficially, becoming an impediment to battery recharging (26). In our series, we did not experience any of these, nor the so-called twiddler's syndrome, in which the patient repeatedly gives his IPG a twiddle, finally leading to extension wire breakage (27). We strongly believe that the risk of these three AEs can be largely reduced by fixating the rIPG in its subcutaneous pocket.

In two patients, the rIPG became overdischarged five times in total, with severe worsening of the OCD symptoms but no suicidality.

\section{Reduction of IPG Replacement Frequency}

In this series of patients with a high frequency of IPG replacement procedures, rIPG usage significantly decreased the frequency of DBS-related surgical procedures in general and of IPG replacement procedures in particular. The rIPGs used have a maximal longevity of nine years (28). In our series, with three patients having a follow-up of more than six years, no IPG needed replacement yet. The future need for rIPG replacement procedures will probably diminish but highly likely not completely take away this surgery frequency reduction.

Besides bringing costs and patient discomfort, IPG replacement inevitably holds an infection risk, which may be higher than during initial hardware implantation. In two retrospective studies the infection rate after IPG replacements was two to three times higher than after the initial DBS procedure $(10 \%$ vs. $3.1 \%$ and $8.7 \%$ vs. $3.7 \%$, respectively) $(29,30)$. Therefore, it seems reasonable that diminishing the frequency of DBS-related surgical procedures, by using rIPGs, decreases the risk of infection. No infections occurred in our series, neither before nor after rIPG implantation.

\section{Limitations}

The major limitations of this study are 1) the relatively small sample size; 2) the lack of a cost analysis; 3 ) the lack of quality-of-life measurements; and 4) that no qualitative question addressing the IPG type preference was asked.

Although we report on only ten patients, this sample size can be rated as considerable given the highly specific population and stringent inclusion criteria.

A cost analysis would have been more convincing for health decision makers than our descriptive report. However, there are three reasons why a cost analysis was not performed. First, the sample size is too small to render reliable result in terms of costs per DALY or QALY when comparing nrlPGs to rIPGs $(31,32)$.

Secondly, as this is a single-center study originating from Belgium, with a very specific health system (entirely publically funded health system, free choice of health providers, total reimbursement for both the IPGs and the implantation surgery, DBS for OCD surgery limited to certain hospitals), any cost analysis resulting from this study would represent the very local Belgian situation (31,32). Lastly, we did not obtain informed consent to collect data such as hospitalization and outpatient clinic visiting frequency in external hospitals or with general practitioners.

We did not include a quality-of-life measurement (e.g., EQ-5D6 (33) or CDC HRQOL-4 (34)), mainly because GAF, HAM-D, and BDI are largely overlapping with these.

A qualitative question on which system (nrlPG or rIPG) the patients preferred would have been valuable, although also very susceptible to recall bias.

\section{CONCLUSIONS}

Based on our series of ten patients, the use of rIPGs in this highly specific psychiatric population appears to be effective, applicable, safe, and capable of reducing the IPG replacement frequency. Therefore, approval and reimbursement of the use of rIPGs for DBS in OCD seems justified. Continued research, based on larger patient samples with longer follow-up is needed to refine which and how DBS for OCD patients can benefit maximally from rIPGs.

\section{Acknowledgements}

We would like to thank Steffen Fieuws of the KU Leuven Statistics Center, the hospital staff, the members of the Committee for Neurosurgery for Psychiatric Disorders, as well as all participating patients and their families.

\section{Authorship Statements}

Bart Nuttin and Lutgardis Gabriëls designed the study. Philippe De Vloo, Simon Raymaekers, Laura Luyten and Lutgardis Gabriëls collected the data. Philippe De Vloo and Kris van Kuyck analyzed the data, which was interpreted by Philippe De Vloo, Laura Luyten, Kris van Kuyck and Bart Nuttin. Philippe De Vloo prepared the manuscript draft with important intellectual input from Kris van Kuyck. All authors critically reviewed the draft and approved the final manuscript.

\section{How to Cite this Article:}

De Vloo P., Raymaekers S., van Kuyck K., Luyten L., Gabriëls L., Nuttin B. 2017. Rechargeable Stimulators in Deep Brain Stimulation for Obsessive-Compulsive Disorder: A Prospective Interventional Cohort Study.

Neuromodulation 2017; E-pub ahead of print. DOl:10.1111/ner.12577

\section{REFERENCES}

1. Stein DJ. Obsessive-compulsive disorder. Lancet 2002;360:397-405. doi:10.1016/ S0140-6736(02)09620-4. 
2. Cosyns $\mathrm{P}$, Gabriels $\mathrm{L}$, Nuttin B. Deep brain stimulation in treatment refractory obsessive compulsive disorder. Verh - K Acad Voor Geneeskd Van Belg 2003;65:385-399400.

3. Nuttin B, Cosyns P, Demeulemeester H, Gybels J, Meyerson B. Electrical stimulation in anterior limbs of internal capsules in patients with obsessive-compulsive disorder. Lancet 1999;354:1526. doi:10.1016/S0140-6736(99)02376-4.

4. Greenberg BD, Gabriels LA, Malone DA, Jr et al. Deep brain stimulation of the ventral internal capsule/ventral striatum for obsessive-compulsive disorder: worldwide experience. Mol Psychiatry 2010;15:64-79. doi:10.1038/mp.2008.55.

5. Lakhan SE, Callaway E. Deep brain stimulation for obsessive-compulsive disorder and treatment-resistant depression: systematic review. BMC Res Notes 2010;3:60. doi:10.1186/1756-0500-3-60.

6. Ooms $\mathrm{P}$, Mantione $\mathrm{M}$, Figee $M$, Schuurman PR, van den Munckhof $\mathrm{P}$, Denys D. Deep brain stimulation for obsessive-compulsive disorders: long-term analysis of quality of life. J Neurol Neurosurg Psychiatry 2014;85:153-158. doi:10.1136/jnnp-2012-302550.

7. Shah RS, Chang S-Y, Min H-K, Cho Z-H, Blaha CD, Lee KH. Deep brain stimulation: technology at the cutting edge. J Clin Neurol Seoul Korea 2010;6:167-182. doi: 10.3988/jen.2010.6.4.167.

8. Blomstedt P, Sjöberg RL, Hansson M, Bodlund O, Hariz MI. Deep brain stimulation in the treatment of obsessive-compulsive disorder. World Neurosurg 2013;80:e245e253. doi:10.1016/j.wneu.2012.10.006.

9. Morishita T, Fayad SM, Goodman WK et al. Surgical neuroanatomy and programming in deep brain stimulation for obsessive compulsive disorder. Neuromodulation $J$ Int Neuromodulation Soc 2014;17:312-319. doi:10.1111/ner.12141.

10. Luyten L, Hendrickx S, Raymaekers S, Gabriëls L, Nuttin B. Electrical stimulation in the bed nucleus of the stria terminalis alleviates severe obsessive-compulsive disorder. Mol Psychiatry 2016;21:1272-1280. doi:10.1038/mp.2015.124.

11. Eldridge $P$, Simpson BA, Gilbart J. The role of rechargeable systems in neuromodulation. Eur Neurol Rev 2011;6:187-192.

12. Harries AM, Major S, Sandhu M, Honey CR. Rechargeable internal neural stimulators-is there a problem with efficacy?. Neuromodulation $J$ Int Neuromodulation Soc 2012;15:214-217-218. doi:10.1111/j.1525-1403.2011.00406.x.

13. O'Rawe JA, Fang $\mathrm{H}$, Rynearson $\mathrm{S}$ et al. Integrating precision medicine in the study and clinical treatment of a severely mentally ill person. PeerJ 2013;1:e177. doi: 10.7717/peerj.177.

14. Ooms $P$, Blankers $M$, Figee $M$ et al. Rebound of affective symptoms following acute cessation of deep brain stimulation in obsessive-compulsive disorder. Brain Stimul 2014;7:727-731. doi:10.1016/j.brs.2014.06.009

15. Vora AK, Ward H, Foote KD, Goodman WK, Okun MS. Rebound symptoms following battery depletion in the NIH OCD DBS cohort: clinical and reimbursement issues. Brain Stimul 2012;5:599-604. doi:10.1016/j.brs.2011.10.004.

16. Goodman WK, Price LH, Rasmussen SA et al. The Yale-Brown obsessive compulsive scale. I. Development, use, and reliability. Arch Gen Psychiatry 1989;46:1006-1011.

17. Hamilton M. The assessment of anxiety states by rating. Br J Med Psychol 1959;32: 50-55.

18. Hamilton M. Rating depressive patients. J Clin Psychiatry 1980:41:21-24.

19. Beck AT, Ward CH, Mendelson M, Mock J, Erbaugh J. An inventory for measuring depression. Arch Gen Psychiatry 1961;4:561-571.

20. Hall RC. Global assessment of functioning. A modified scale. Psychosomatics 1995; 36:267-275. doi:10.1016/S0033-3182(95)71666-8.
21. Medtronic Manuals Hypersearch. n.d. October 7. 2015. https://manuals.medtronic. $\mathrm{com} / \mathrm{manuals} / \mathrm{search}$ ?region $=$ us\&cfn $=8870$ AAR01\&lang $=$ en\&manuallanguage $=\mathrm{en} \&$ manualType $=$ Programming + Guide\&therapy $=D B S+$ for + Parkinsons.

22. Waln $\mathrm{O}$, Jimenez-Shahed J. Rechargeable deep brain stimulation implantable pulse generators in movement disorders: patient satisfaction and conversion parameters. Neuromodulation J Int Neuromodulation Soc 2014;17:425-430. doi:10.1111/ner.12115.

23. Timmermann $L$, Schüpbach $M$, Hertel $F$ et al. A new rechargeable device for deep brain stimulation: a prospective patient satisfaction survey. Eur Neurol 2013;69:193199. doi:10.1159/000342236.

24. Fakhar K, Hastings E, Butson CR, Foote KD, Zeilman P, Okun MS. Management of deep brain stimulator battery failure: battery estimators, charge density, and importance of clinical symptoms. PLoS One 2013;8:e58665. doi:10.1371/ journal.pone.0058665.

25. Ondo W, Hewgley T, Almaguer M. Interesting complication of the Medtronic Activa RC impulse pulse generator. Mov Disord off J Mov Disord Soc 2012;27:333-334. doi: $10.1002 /$ mds.24019.

26. Chelvarajah $R$, Lumsden $D$, Kaminska $M$ et al. Shielded battery syndrome: a new hardware complication of deep brain stimulation. Stereotact Funct Neurosurg 2012; 90:113-117. doi:10.1159/000336342.

27. Burdick AP, Okun MS, Haq IU et al. Prevalence of Twiddler's syndrome as a cause of deep brain stimulation hardware failure. Stereotact Funct Neurosurg 2010;88:353359. doi:10.1159/000319039.

28. Activa RC Neurostimulator for Deep Brain Stimulation | Features and Specifications n.d. December 16. 2015. http://professional.medtronic.com/pt/neuro/dbs-md/prod/ activa-rc/features-specifications/index.htm\#.U3X7KiirMxQ.

29. Pepper J, Zrinzo L, Mirza B, Foltynie T, Limousin P, Hariz M. The risk of hardware infection in deep brain stimulation surgery is greater at impulse generator replacement than at the primary procedure. Stereotact Funct Neurosurg 2013;91:56-65. doi: $10.1159 / 000343202$

30. Bhatia S, Oh M, Whiting T, Quigley M, Whiting D. Surgical complications of deep brain stimulation. A longitudinal single surgeon, single institution study. Stereotact Funct Neurosurg 2008;86:367-372. doi:10.1159/000175799.

31. Generalized Cost-Effectiveness Analysis. WHO. October 29. 2016. http://www.who. int/choice/cost-effectiveness/generalized/en/.

32. Jamison DT, Breman JG, Measham AR et al. Cost-effectiveness analysis. October 29. 2016. The International Bank for Reconstruction and Development/The World Bank, 2006. https://www.ncbi.nlm.nih.gov/books/NBK10253/.

33. Herdman M, Gudex C, Lloyd A et al. Development and preliminary testing of the new five-level version of EQ-5D (EQ-5D-5L). Qual Life Res Int J Qual Life Asp Treat Care Rehabil 2011;20:1727-1736. doi:10.1007/s11136-011-9903-x.

34. Moriarty DG, Zack MM, Kobau R. The Centers for Disease Control and Prevention's Healthy Days Measures - population tracking of perceived physical and mental health over time. Health Qual Life Outcomes 2003;1:37. doi:10.1186/1477-7525-1-37.

\section{SUPPORTING INFORMATION}

Additional supporting information may be found online in the supporting information tab for this article. 